\title{
Efectos de un Programa de Rehabilitación Cardiaca Fase I en Pacientes Revascularizados
}

\section{Effects of Cardiac Rehabilitation in Patients Revascularized Phase I}

\author{
Recibido: 14 Mayo 2014/Enviado para modificación: 2 de Julio 2014/Aceptado: 23 de Octubre 2014
}

\author{
Lilian Barros Higgins ${ }^{1}$ \\ Clínica de la Costa \\ Leynis Osorio Álvarez ${ }^{2}$ \\ Fundación Policlínica de Ciénaga
}

Kelly Navarro García ${ }^{3}$

Clínica de la Costa

Milagros de Avila Meza ${ }^{4}$

Clínica del Prado

Rosmeri Acosta Barrios 5

Clínica Centro

\section{RESUMEN}

Introducción: Las enfermedades cardiovasculares son una de las primeras causas de muerte a nivel mundial. Es así como los programas de rehabilitación cardiaca fase I han ido evolucionando y se considera como una de las primeras estrategias utilizadas para disminuir el riesgo de reincidencia de un evento coronario. Objetivo: describir la efectividad de la rehabilitación cardiaca fase I, en un grupo de pacientes pre y postquirúrgicos de revascularización miocárdica. Materiales y Métodos: Estudio cuasi experimental. Se aplicó con la escala de Borg y la caminata de los 6 minutos a 10 pacientes pre y post operatorio de revascularización miocárdica en un programa de rehabilitación cardiaca fase I en la Clínica de la Costa. Los datos se analizaron en el programa SPSS. Resultados: La hipertensión es la patología más frecuente con un $80 \%$ de la población estudiada un 70\% presentó un riesgo cardiovascular moderado; el factor de riesgo que predominó fue la inactividad física con un 70\% se encontró una diferencia significativa según el predicho de Troosther de 405 metros, recorrido en comparación con la primera evaluación así mismo al inicio del programa según la escala de Borg el esfuerzo percibido fue 5,5 puntos y al finalizarlo se obtuvo un promedio de 1.8 puntos, que corresponde a un esfuerzo suave. Conclusión: con base a los resultados, clínicos el impacto que tuvo la siguiente investigación fue demostrar con la implementación de la fase 1 de los programas de rehabilitación cardiaca que los pacientes sometidos a este estudio mejoraron la capacidad funcional y tolerancia al ejercicio.

Palabras Clave:Revascularización, enfermedad coronaria, factores de riesgo, ejercicio, rehabilitación (Fuente: DeCS).

\section{ABSTRACT}

Introduction: Cardiovascular diseases are one of the leading causes of death worldwide. I show programs phase I cardiac rehabilitation have been evolving and is considered as one of the first strategies used to decrease the risk of recurrence of a coronary event. Objective: To describe the effectiveness of phase I cardiac rehabilitation in a group of pre postsurgical patients and myocardial revascularization. Methods: Quasi-experimental study. Was applied to the scale of Borg and walk 6 minutes to 10 patients pre and post-operative myocardial revascularization in cardiac rehabilitation program in clinical phase I of the coast. Data were analyzed in SPSS. Results: Hypertension is the most frequent pathology with $80 \%$ of the study population $70 \%$ had a moderate cardiovascular risk ; the risk factor was predominant physical inactivity with $70 \%$ a significant difference according to the predicted Troosther of 405 meters was found, driven compared to the first evaluation likewise at the beginning of the program as the Borg scale was perceived exertion 5.5 points or after completing an average of 1.8 points, corresponding to a soft effort was obtained. Conclusion: based on the results, the clinical impact of the following study was to demonstrate the implementation of phase 1 of the cardiac rehabilitation programs for patients undergoing this study improved functional capacity and exercise tolerance.

Keywords:revascularization, coronary disease risk factors, exercises rehabilitation.(Source: MeSH, NLM).

Para citar este artículo: Barros L, Osorio L, Navarro K, De Avila M, Acosta R. Efectos de un Programa de Rehabilitación Cardiaca Fase I en Pacientes Revascularizados.Cienc. innov. salud. 2014; 2 (1):76-81

${ }^{1}$ Fisioterapeuta. Especialista en Adaptación y rehabilitacióncardiopulmonar y vascular. Clínica de la Costa. Correoelectrónico: lbarros@

${ }^{2}$ Fisioterapeuta. Especialista en Adaptación y rehabilitacióncardiopulmonar y vascular. Fundaciónpoliclínicaciénaga. fta.leynis@ @ hotmail.com

${ }^{3}$ Fisioterapeuta. Especialista en Adaptación y rehabilitacióncardiopulmonar y vascular. Clínica de la Costa. Barranquilla. Correo: kelly_navafisio@ hotmail.com

${ }^{4}$ Fisioterapeuta. Especialista en Adaptación y rehabilitación cardiopulmonar y vascular. Clínica del Prado. Barranquilla. Correo: milagrosdeavila@ hotmail.com

${ }^{5}$ Fisioterapeuta. Especialista en Adaptación y rehabilitación cardiopulmonar y vascular. Clínica Centro. Barranquilla. Correo: romeryacosta25@ hotmail.com 


\section{Introducción}

El crecimiento de la población y el aumento en la esperanza de vida están conduciendo a un rápido aumento del número total de adultos de mediana edad y mayores y por consiguiente al incremento de las cifras de muertes por enfermedades crónicas no trasmisibles $(1,2$,) de acuerdo con el Informe del Estado Global en Salud de la Organización Mundial de la Salud (OMS) publicado en abril de 2011, las enfermedades crónicas no transmisibles fueron la causa de aproximadamente el $63 \%$ del total de muertes ocurridas en el mundo en el año 2008, siendo las cardiovasculares $29,8 \%$ las de mayor incidencia (3).

En Colombia predomina la enfermedad coronaria, en los hombres predomina la enfermedad cardiaca isquémica en hombres $\mathrm{y}$ en las mujeres las enfermedades relacionadas con la hipertensión arterial, como la enfermedad cerebro vascular y la insuficiencia cardiaca; estas enfermedades constituyen problemas importantes de salud pública en el país $(4,5)$.

La enfermedad coronaria es la causa individual más frecuente de muerte en el mundo. Más de 7 millones de personas mueren cada año como consecuencia de la cardiopatía isquémica, lo que corresponde a un $12,8 \%$ de todas las muertes (6). La enfermedad coronaria multivaso es una patología en crecimiento que tiene importantes repercusiones en cuanto a morbilidad y mortalidad en países desarrollados y en vía de desarrollo; para el año 2003, la enfermedad coronaria causó una de cada cinco muertes en Estados Unidos y es la principal causa de muertes en hombres y mujeres en ese país (7).

Los pacientes que sobreviven a un evento isquémico agudo tienen más riesgo de padecer otro evento a corto plazo, por lo tanto, deben aplicarse estrictas medidas de prevención secundaria; así, los procedimientos de revascularización como cateterismo o cirugía se aplican en proporción cada vez mayor a pacientes que han padecido a un evento cardiovascular, en los cuales la cirugía de revascularización Miocárdica es la más frecuente de las cirugías cardíacas y uno de los mayores logros quirúrgicos desde el siglo pasado (3).

Dada la gradual incidencia de las EC y el creciente acceso a los procedimientos quirúrgicos en Colombia, se hacen necesaria la realización de estudios investigativos que muestren la evaluación clínica de estos pacientes que son sometidos a revascularización percutánea o quirúrgica. Este tipo de procedimiento genera en el paciente un impacto en su estado físico, social y laboral, por el tiempo prolongado que deben permanecer en decúbito prolongado, disminuyendo su condición física, que se manifiesta por la presencia de taquicardia refleja durante la actividad física, hipotensión ortostática, disminución de la masa muscular, descalcificación ósea y trastornos de la movilidad articular, entre otras (8).

La revascularización Miocárdica a pesar de ser una opción quirúrgica que ofrece prolongar la vida de un individuo con enfermedad coronaria, acarrea una serie de complicaciones que de no ser manejadas o controladas a tiempo alteran el funcionamiento fisiológico normal de algunos sistemas como el pulmonar, musculo esquelético, cardiovascular, entre otros, afectando el desempeño físico, laboral, social y emocional del individuo $(9,10)$.

El entrenamiento con ejercicio físico aumenta el consumo de oxígeno máximo (VO2) y la capacidad física, contribuyendo a mejorar la calidad de vida y el pronóstico en pacientes con falla cardiaca, logrando adaptaciones en la estructura y función del músculo esquelético, el flujo sanguíneo periférico y la función endotelial; asimismo, incrementa el tono vagal, disminuye el tono simpático y el riesgo de arritmias (11).

En este sentido, la rehabilitación cardiaca (RC) se convierte en la intervención que posibilita mejorar la condición física del paciente sometido a revascularización coronaria, permitiendo una disminución de la frecuencia cardiaca y de las cifras de presiones sistémicas, sistólica y media durante el ejercicio y el reposo (12). Por ello, un programa de RC fase I modificaría de manera positiva la capacidad funcional de las personas, ayudando al paciente a reintegrarse rápidamente a sus actividades de la vida diaria, mejorando la actitud del sujeto frente a su evento, reduciendo la estancia hospitalaria y los costos de la entidad prestadora de salud $(12,13)$.

El objetivo del presente estudio es describir la efectividad de la rehabilitación cardiaca fase I en un grupo de pacientes post quirúrgicos de revascularización Miocárdica.

\section{Materiales y Métodos}

Se realizó un estudio cuasi experimental en personas con enfermedad coronaria de vasos. Se seleccionaron 10 pacientes del total ingresados a la Clínica de la Costa durante el periodo de septiembre a noviembre del 2013; los participantes fueron personas de ambos 
sexos y diferentes grupos de edad con diagnóstico médico de enfermedad coronaria de vasos incluidos en el protocolo de cirugía cardiovascular de la institución de salud para cirugía de revascularización Miocárdica.

Para el presente proyecto se excluyeron los pacientes que tuvieran lesión de tronco por alto riesgo de muerte súbita, valvulopatias, con enfermedad cerebro vascular y con inestabilidad hemodinámica.

Previo consentimiento informado, se evaluó la capacidad cardiorespiratoria mediante la Prueba de Caminata de los 6 Minutos, la cual se realizó a todos los pacientes el día de ingreso a la clínica. Se efectuó la prueba en un sitio absolutamente plano con una longitud igual a 30 metros, no transitado, donde el paciente realizó la mayor cantidad de vueltas por el tramo delimitado en $6 \mathrm{~min}$. Se registró la percepción subjetiva de disnea y fatiga de piernas a través de la Escala de Borg. Igualmente, se midió la saturación de oxígeno, y la frecuencia cardíaca y respiratoria antes y después de la prueba.

Se revisaron las historias clínicas de los sujetos participantes en el estudio, registrando el diagnóstico médico, las características socio demográficas, sexo, estado civil, nivel de escolaridad, aseguramiento. La anterior evaluación se realizó el día de ingreso y de egreso a la clínica; el periodo de hospitalización de los pacientes fue de 15 días, cinco antes de cirugía y 10 después de cirugía.

A cada paciente se le aplicó el programa de rehabilitación cardiaca fase I dos veces al día durante su estadía en la institución de salud. El protocolo de intervención en rehabilitación cardiaca para pacientes pre y pos-quirúrgicos cardiacos fase I estuvo constituido por los siguientes componentes $(14,15,16)$ :

- Educación para la salud y prevención de su enfermedad: referente a su procedimiento quirúrgico, protocolo desde el momento de salida de su habitación en hospitalización hasta su entrada a la Unidad de Cuidado Intensivo y recomendaciones a seguir para después de su cirugía.

- Incentivo respiratorio: realizando 3 series de 10 repeticiones cada 2 horas durante el día.

- Ejercicios de expansibilidad torácica: 3 series de 5 repeticiones 4 veces al día.

- Respiración combinada con cinesiterapia de miembros superiores e inferiores: ejercicios activos libres en compañía de ejercicios respiratorios. Realizaban 3 series de 10 repeticiones 2 veces al día.

- Movilizaciones Diafragmáticas: respiración abdominal diafragmática en 3 series de 20 repeticiones, ejercicios con los labios fruncidos para realizar en 2 series de 20 repeticiones.

- Educación postural: antes de la cirugía se realizaba un ensayo de su posición en la cama, el cambio de decúbito a posición sedente, de sedente a colocarse de pie, de pie a sentarse en una silla, de pie a acostarse después de la cirugía.

\section{Resultados}

En cuanto a las características socio demográficas de los pacientes que participaron en el estudio, 5 de los pacientes fueron mujeres y 5 hombres entre los 52 y 68 años de edad. Todos los sujetos de estudio tienen una pareja permanente y la mayoría tiene un nivel de escolaridad baja (60\%) y de estrato socioeconómico 1 (70\%). El $80 \%$ (8) de la población pertenece al régimen de seguridad social subsidiado (Tabla 1 ).

Tabla 1. Características socio demográficas de los pacientes atendidos

\begin{tabular}{llcc}
\hline \multicolumn{1}{c}{ Variables } & \multicolumn{1}{c}{ Nivel } & Frec. Obs & Frec, \% \\
\hline \multirow{2}{*}{ Sexo } & Femenino & 5 & 50 \\
& Masculino & 5 & 50 \\
\hline Estrato & Estrato 1 & 7 & 70 \\
socio- & Estrato 2 & 2 & 20 \\
económico & Estrato 3 & 1 & 10 \\
\hline \multirow{3}{*}{ Nivel } & Iletrado & 2 & 20 \\
escolaridad & Primaria & 4 & 40 \\
& Secundaria & 3 & 30 \\
& Técnico & 1 & 10 \\
\hline Tipo de & Contributivo & 1 & 10 \\
seguridad & Subsidiado & 8 & 80 \\
social & Vinculado & 1 & 10 \\
\hline \multirow{2}{*}{ Edad } & $\bar{x} \pm s d$ & $61(5,4)$ años & $52-68$ \\
& & & años \\
\hline \multirow{2}{*}{ Fuente: } &
\end{tabular}

Fuente: Historias Clínicas y Valoración de Rehabilitación Cardiaca.

La hipertensión arterial es la patología más frecuente dentro de la población estudiada $(8 ; 80 \%)$. Un $70 \%$ (7) de los pacientes estudiados tiene un riesgo cardiovascular moderado. Se identificó que de los 10 pacientes estudiados el factor de riesgo que predomina es la inactividad física con (70\%) (Tabla 2). 
Tabla 2. Características de salud de los sujetos de estudio

\begin{tabular}{llcc}
\hline Variables & Nivel & Frec. Obs. & Frec. \% \\
\hline Estratificación & Riesgomoderado & 7 & 70 \\
del riesgo & Riesgo alto & 3 & 30 \\
\hline \multirow{3}{*}{$\begin{array}{l}\text { Patologías } \\
\text { asociadas }\end{array}$} & Hipertensión & 10 & 100 \\
& Diabetes tipo 2 & 2 & 50 \\
& Enfermedad & 8 & 80 \\
\hline \multirow{3}{*}{$\begin{array}{l}\text { Factores de } \\
\text { riesgo }\end{array}$} & Inactividadfísica & 7 & 70 \\
& Obesidad & 3 & 30 \\
\hline & Consumo de & 3 & 30 \\
\hline
\end{tabular}

Fuente: Historias Clínicas y Valoración de Rehabilitación Cardiaca.

Se encontró diferencias significativas $(\mathrm{p}<0,05)$ entre los valores medios obtenidos al inicio y al final del programa de RC fase I. La distancia media según el predicho de Trooster fue de 595 metros y la recorrida durante la primera evaluación de $189 \mathrm{mt}$ y luego del programa de RC fue de 594metros. Igualmente, sucedió con esfuerzo percibido por los paciente durante la prueba de Caminata de los 6 minutos, el promedio al ingresar a la institución de salud fue de 5,5 puntos, que según la Escala Borg del esfuerzo percibido es una sensación de esfuerzo fuerte y al final del programa de RC fase I, se obtuvo un promedio de 1,8 puntos, que corresponde a un esfuerzo suave. El promedio de minutos recorridos durante la primera evaluación fue de 3 minutos y al final del programa fue de 6 minutos (Tabla 3 ).

Tabla 3. Medias de los metros recorridos, el esfuerzo percibido y los minutos caminando

\begin{tabular}{|c|c|c|c|c|}
\hline Variables & $\begin{array}{l}\text { Medias } \\
\text { Pre- } \\
\text { evaluació } \\
\mathrm{n}\end{array}$ & $\begin{array}{l}\text { Medias } \\
\text { post- } \\
\text { evaluació } \\
\mathrm{n}\end{array}$ & $\begin{array}{l}\text { Diferencia } \\
\text { s de } \\
\text { medias }\end{array}$ & $\begin{array}{l}\text { Valo } \\
\text { r de } \\
\mathrm{p}\end{array}$ \\
\hline Metros recorridos & 189 (114) & $594(61)$ & -405 & $\begin{array}{l}0,00 \\
0\end{array}$ \\
\hline Esfuerzopercibido & $5,5(1,2)$ & $1,8(0,9)$ & 3,7 & $\begin{array}{l}0,00 \\
0\end{array}$ \\
\hline $\begin{array}{l}\text { Minutoscaminand } \\
\mathrm{o}\end{array}$ & $3(1)$ & $6(0,1)$ & -3 & $\begin{array}{l}0,00 \\
0\end{array}$ \\
\hline
\end{tabular}

\section{Discusión}

Se observa en el presente estudio que todos los participantes tienen como factor de riesgo la hipertensión arterial; estos resultados coinciden con un estudio de casos y controles donde realizan un análisis univariado entre grupos y por factor de riesgo, donde el $88,3 \%$ de los casos tuvieron HTA y en los controles fue de 73,3\% manteniéndose como el factor de riesgo más prevalente. En el caso de la HTA, casi triplica el riesgo de enfermedad grave cuando se presenta aisladamente, aunque al asociarse a antecedentes familiares de EC grave, sextuplica este riesgo respecto de los pacientes que no tienen antecedentes familiares; ambos factores de riesgo, diabetes e HTA se mostraron asociados a la presencia de enfermedad ateromatosa coronaria subclínica, en individuos menores a 45 años (17). Se ratifica una vez más que la hipertensión arterial es una patología asociada a enfermedades cardiovasculares.

Los resultados obtenidos durante esta investigación demostraron que el factor de riesgo más relévate fue la inactividad física. Este hallazgo coincide con los del estudio realizado en la ciudad de Medellín donde reportan que solo una de cada cinco personas realiza suficiente actividad física para proteger la salud, consolidándose este factor de riesgo como uno de los principales pre disponentes para la aparición precoz de las enfermedades cardiovasculares (18).

Estudios anteriores son consistentes con nuestra investigación reportando un incremento en la prueba de caminata de 6 minutos después de un programa de rehabilitación cardiaca; los incrementos reportados en la literatura de la distancia recorrida en la Prueba de Caminata de 6 minutos después del programa van desde un $15 \%$ hasta un $33 \%$; en promedio los pacientes mejoraron la distancia en la prueba de 6 minutos 139 metros, pasando de $404 \pm 99$ a $543 \pm 93$ metros $(\mathrm{p}<0.001)$ o el equivalente a $34 \%$ más en la distancia recorrida después del PRC(19).

Otros autores han reportado que los pacientes postoperados de revascularización Miocárdica mejoraron la distancia durante la prueba de caminata de los 6 minutos después de un programa de rehabilitación cardiaca fase I (20); en un grupo de 425 pacientes se registró un aumento promedio de $21,6 \%$ en la distancia recorrida después del PRC, incrementando 73,9 metros en la distancia al caminar (21).

En otro estudio realizado en pacientes con insuficiencia cardiaca se reportó un aumento de 17,1\% en la distancia de la Prueba de Caminata de 6 minutos después de la rehabilitación cardiaca, lo cual representó un incremento de 75 metros (19). Se ratifica una vez más que los programas de rehabilitación cardiaca muestran buenos resultados aplicándose desde fase I ante de un procedimiento cardiovascular 
mejorando notablemente su capacidad funcional $\mathrm{y}$ tolerancia al ejercicio.

Con este estudio se ratifica una vez más que la prueba de caminata de 6 minutos es un método factible y fiable para medir la capacidad física de los pacientes pertenecientes en un Programa de Rehabilitación Cardiaca con ejercicio supervisado y monitorizado; ya que logra una mejoría de la Capacidad Funcional incluso en los Pacientes con Fracción de Eyección muy deprimida; La intervención fisioterapeuta ha participado activamente en el proceso de rehabilitación del paciente con enfermedad coronaria con el objetivo de brindar por medio de la actividad física temprana una mejoría de las funciones cardiopulmonares y osteomusculares realizando una adecuada prescripción del ejercicio y logrando de esa forma que el paciente tenga una adecuada adaptación postoperatoria para continuar con su recuperación mejorando su capacidad funcional y una pronta reinserción en sus actividades de la vida diaria.

\section{Referencias Bibliográficas}

1. Organización Mundial de la Salud. Estadísticas Sanitarias 2012. Ginebra; 2012.

2. Campos AM, Moya PD, Mendoza MJ, Duran NE. Las enfermedades crónicas no transmisibles y el uso de tecnologías de información y comunicación: revisión sistemática. RevCuid. 2014; 5 (1):661-669.

3. Gómez L. Las enfermedades cardiovasculares: un problema de salud pública y un reto global. Biomédica. 2011; 31(4): 467-473.

4. Pastor VM. Enfermedades cardiovasculares y atención primaria en salud. Rev Salud Unisucre. 2013; 1(2):134-138.

5. Rodríguez BS, Almeida GJ, Pérez GJ. Enfermedad coronaria multivaso, disfunción endotelial y angioplastia en la diabetes mellitus. A propósito de un caso. CorSalud. 2014; 6(1):110-118.

6. Ibáñez J, Riera M, Sáez IJ, Amezaga R, Tarrío R, Campillo $\mathrm{C}$, et al. Factores de riesgo de la supervivencia a largo plazo de la cirugía coronaria aislada. Medicina Balear. 2014; 29(1):19-24.

7. Fernández A. Angioplastia con stent vs. cirugía de revascularización coronaria en enfermedad multivaso. Rev.MD.Colom.Cardiol. 2009; 16(2):53-63.
8. Cruz BL. Impacto de la rehabilitación cardiaca intrahospitalaria en pacientes con IMA. Rev Ciencias Médicas. 2006; 10(3): 81-90.

9. Sousa LC. Fisioterapia intensiva. Bogota: Distribuna; 2012.

10.Godinho A, Alves A, Pereira A, Santos P T. Cirugía de revascularización miocárdica con circulación extracorpórea versus sin circulación extracorpórea: un Metanálisis. ArqBrasCardiol. 2012; 98(1):87-94.

11.Atehortúa SD, Gallo J, Rico M, Durango L. Efecto de un programa de rehabilitación cardiaca basado en ejercicio sobre la capacidad física, la función cardiaca y la calidad de vida, en pacientes con falla cardiaca. Rev.Col.Cardiol. 2011; 18(1) 25-36.

12.Báez L, Camacho P, Abreu P, Arenas I, Jaimes G. La importancia de la rehabilitación cardiovascular: experiencia en la Fundación Cardiovascular de Colombia. Rev.Col.Cardiol. 2006; 13(2):90-96.

13.Hernández GS, MustelierOJ, Rivas EE. Fase hospitalaria de la rehabilitación cardíaca. Protocolo para el síndrome coronario agudo. Cor Salud. 2014; 6(1):97-104.

14.Borghi AS, Mendes RG, Costa FM, Di Lorenzo VA, Oliveira CR, Luzzi S. The influences of positive end expiratory pressure (PEEP) associated with physiotherapy intervention in phase I cardiac rehabilitation. Clinics. 2005; 60 (6): 465-472.

15.Matsunaga A, Masuda T, Ogura MN, Saitoh M, Kasahara Y, Iwamura T, et al. Adaptation to low intensity exercise on a cycle ergometer by patients with acute myocardial infarction undergoing, phase i cardiac rehabilitation. Circ J. 2004; 68 (10): 938945.

16.Ennio B, Sanchez B. Physical therapy in phase I of a cardiac rehabilitation program for patients with acute coronary syndrome. Bioline international. 2009; 0 (39): 26-33.

17. Olavegogeascoechea P, Urdiales P, Schroeder A, López C A, Cari M, García P. Factores de riesgo clásicos asociados a la enfermedad coronaria severa. Un estudio de casos y controles argentino. Europea ScientificJournal. 2014; 10(15):490-501.

18. Martínez L, Saldarriaga J, ÉulerSF. Actividad física en Medellín: desafío para la promoción de la salud. Rev. FacNac Salud Pública. 2008; 26(2): 117-123.

19.Araya RF, Ureña BP, Sánchez UB, Blanco RL, Rodríguez MA, Moraga RC. Influencia de la capacidad funcional Inicial en marcadores fisiológicos después de un programa de 
rehabilitación cardiaca. Rev. Costarric. Cardiol. 2013; 15(1): 5-11.

20.Araya R, Briggs KK, Bishop SR, Miller CE, MoncadaJJ, Grandjean PW. Who is likely to benefit from phase II cardiac rehabilitation? CardiopulmRehabil Prev. 2010; 30(2): 93-100.

21.Listerman J, Bittner V, Sanderson BK, Brown TM. Cardiac rehabilitation outcomes: impact of co morbidities and age. CardiopulmRehabil Prev. 2011; 31(6):342-348. 